\title{
Protocol for a pilot randomized controlled feasibility study of brief interpersonal psychotherapy for addressing social- emotional needs and preventing excess gestational weight gain in adolescents
}

Lauren B. Shomaker ${ }^{1,2,3^{*}+}$, Lauren D. Gulley ${ }^{1,3+}$, Emma L. M. Clark ${ }^{1,3}$, Allison M. Hilkin ${ }^{3}$, Bernadette Pivarunas ${ }^{1}$, Marian Tanofsky-Kraff', Kristen J. Nadeau ${ }^{3}$, Linda A. Barbour ${ }^{5,6}$, Stephen M. Scott ${ }^{3,6}$ and Jeanelle L. Sheeder ${ }^{3,6}$

\begin{abstract}
Background: Excess gestational weight gain (GWG) in pregnant adolescents is a major public health concern. Excess GWG increases risk of pregnancy complications as well as postpartum and offspring obesity and cardiometabolic disease. Prevention interventions for pregnant adults that target lifestyle modification (i.e., healthy eating/physical activity) show insufficient effectiveness. Pregnant adolescents have distinct social-emotional needs, which may contribute to excess GWG. From an interpersonal theoretical framework, conflict and low social support increase negative emotions, which in turn promote excess GWG through mechanisms such as overeating and physical inactivity.

Methods: The current manuscript describes the design of a pilot randomized controlled feasibility trial of adolescent interpersonal psychotherapy (IPT) to address social-emotional needs and prevent excess GWG. Up to 50 pregnant, healthy adolescents 13-19y, 12-18 weeks gestation are recruited from an interdisciplinary adolescent maternity hospital clinic and randomized to IPT + usual care or usual care alone. IPT involves 6 individual 60-minute sessions delivered by a trained behavioral health clinician during 12-30 weeks gestation. Sessions include relationship psychoeducation, emotion identification and expression, and teaching/role-playing communication skills. Between sessions, adolescents are instructed to complete a daily journal and to have conversations to work on relationship goals. Outcomes are assessed at baseline, mid-program, post-program, and 3-months postpartum. Primary outcomes are feasibility and acceptability based upon rate of recruitment, session attendance, program acceptability ratings, and follow-up retention. Secondary outcomes are perinatal social functioning, stress, depression, and eating behaviors assessed with validated surveys and interviews; perinatal physical activity and (Continued on next page)
\end{abstract}

\footnotetext{
* Correspondence: lauren.shomaker@colostate.edu

${ }^{\dagger}$ Lauren B. Shomaker and Lauren D. Gulley contributed equally to this work.

'Department of Human Development and Family Studies, Colorado State

University, 1570 Campus Delivery, Fort Collins, CO 80523-1570, USA

${ }^{2}$ Department of Community and Behavioral Health, Colorado School of

Public Health, Aurora, CO, USA

Full list of author information is available at the end of the article
}

C C The Author(s). 2020 Open Access This article is licensed under a Creative Commons Attribution 4.0 International License, which permits use, sharing, adaptation, distribution and reproduction in any medium or format, as long as you give appropriate credit to the original author(s) and the source, provide a link to the Creative Commons licence, and indicate if changes were made. The images or other third party material in this article are included in the article's Creative Commons licence, unless indicated otherwise in a credit line to the material. If material is not included in the article's Creative Commons licence and your intended use is not permitted by statutory regulation or exceeds the permitted use, you will need to obtain permission directly from the copyright holder. To view a copy of this licence, visit http://creativecommons.org/licenses/by/4.0/ The Creative Commons Public Domain Dedication waiver (http://creativecommons.org/publicdomain/zero/1.0/) applies to the data made available in this article, unless otherwise stated in a credit line to the data. 


\begin{abstract}
(Continued from previous page)
sleep measured via accelerometer; GWG from measured weights; and at 3-months postpartum only, maternal adiposity by dual energy $\mathrm{x}$-ray absorptiometry, maternal insulin sensitivity derived from 2-hour oral glucose tolerance testing, and infant adiposity by air displacement plethysmography.

Discussion: This pilot trial will address a key gap in extant understanding of excess GWG prevention for a high-risk population of adolescents. If feasible and acceptable, brief psychotherapy to address social-emotional needs should be tested for its effectiveness to address excess GWG and postpartum maternal/infant health. If effective, such an approach has potential to interrupt an adverse, intergenerational cycle of social-emotional distress, obesity, and cardiometabolic disease among young mothers and their offspring.
\end{abstract}

Trial registration: ClinicalTrials.gov NCT03086161, retrospectively registered

Keywords: Interpersonal psychotherapy, Depression, Obesity, Pregnancy, Adolescence

\section{Background}

An estimated 194,400 babies were born in 2017 to mothers ages 15-19 years in the USA [1]. Major racial/ ethnic and socioeconomic health disparities persist in adolescent pregnancy and childbearing. Non-Hispanic Black, Hispanic, and American Indian/Alaska Native adolescents are far more likely to bear children (27-33 per 1000) than Non-Hispanic White (13 per 1000) and Asian adolescents (3 per 1000) [1]. Similar to adults, the majority of pregnant adolescents, approximately twothirds, gain more weight in pregnancy than recommended by the Institute of Medicine guidelines [2]. This excess weight gain pattern is observed both in adolescents who are lean and those who have overweight/obesity. Moreover, high rates of excess gestational weight gain (GWG) remain consistent when based upon adultcutoffs for pre-pregnancy body mass index (BMI; $\mathrm{kg} / \mathrm{m}^{2}$ ) or on age- and sex-adjusted pre-pregnancy BMI $(z$ score) [2]. Experts have called for recognition of excess GWG in adolescent pregnancy as a major public health concern because it not only increases antepartum and peripartum complications but also increases the risk of postpartum weight retention and future metabolic risk for both the mother and her offspring [3].

\section{Problem of excess GWG in adolescent pregnancy}

Excess GWG in adolescent pregnancy has multiple negative consequences. Excess GWG amplifies the risk of pregnancy complications from pre-existing obesity, including gestational diabetes, hypertension, preeclampsia, and need for Cesarean delivery [4-8]. In addition, excess GWG amplifies mothers' risk of obesity and cardiometabolic disease after pregnancy [9-12]. The more excess weight gained in pregnancy, the more weight retained in the postpartum, increasing mothers' long-term risk of obesity and cardiometabolic disease progression [9-12]. In a large investigation of maternal and fetal growth during adolescent pregnancy, adolescents with excess GWG had greater postpartum weight retention, BMI, central adiposity, and peripheral adiposity over a 2-year period compared to adolescents who gained recommended amounts of weight [13]. Pregnant adolescents with excess GWG were nearly 5 times more likely to have obesity approximately 12 years post-delivery than those who gained appropriate weight [9]. While similar adverse effects have been documented in adults [14], excess GWG in adolescent pregnancy may be particularly deleterious [13]. In a study comparing adolescent and adult pregnancies, adolescents with excess GWG continued to gain more weight between an initial and subsequent pregnancy as compared to adults [15]. Adolescence itself is marked by increases in weight and adiposity in females; therefore, pubertal gains in central adiposity are likely exacerbated when pregnancy is timed in adolescence [13].

Beyond the adverse effects on mothers, excess GWG also affects offsprings' risk of obesity and cardiometabolic disease $[7,8,16-21]$. This intergenerational effect perpetuates a cycle of obesity and cardiometabolic disease in a population at high-risk for social stressors, emotional difficulties, obesity, and preventable chronic illness [22-25]. Offspring of adolescent and adult mothers with excess GWG have higher odds of macrosomia, greater neonatal adiposity, higher BMI in childhood and adolescence, and greater cardiovascular disease risk factors in childhood [7, 8, 16-21, 26]. The "fetal overnutrition" explanation, supported by animal and human studies, is that intrauterine caloric excess creates a metabolic milieu that heightens obesity and cardiometabolic risk, independent of other genetic and postnatal environmental risk factors, through a host of mechanisms such as insulin resistance and subsequent hyperinsulinemia, hyperglycemia, hypertriglyceridemia, increased fat deposition in subcutaneous and intrahepatic depots, changes in appetite regulation, mitochondrial function, impaired functioning of the adipoinsular axis, and epigenetic modifications [27]. Thus, feasible and effective prevention of excess GWG in pregnant adolescents is a priority for reducing adolescent mothers' obesity and cardiometabolic disease risk, and for disrupting the transgenerational cycle of obesity and cardiometabolic disease affecting offspring of successive generations [28]. 


\section{Prevention of excess GWG}

There have been few interventions designed to address excess GWG in pregnant adolescents. In adult pregnant women, the standard approach is lifestyle modification intended to promote healthy eating and physical activity. In an expert review of over 50 randomized controlled trial studies in pregnant adult women, existing programs unfortunately were concluded to be costly, time intensive, and limited in effectiveness and scope [29]. In nonpregnant adolescents who have overweight/obesity, lifestyle-based interventions to address weight management face challenges for adherence and effectiveness, particularly in youth from historically disadvantaged racial/ethnic groups [30-34]. Thus, alternative strategies to lifestyle-based approaches that are feasible and acceptable to pregnant adolescents are highly warranted.

One potentially promising framework is to target the underlying social-emotional contributors to excess GWG in pregnant adolescents. Interpersonal conflict, insufficient social support, and emotional concerns such as depression are highly prevalent in pregnant adolescents [35]. During pregnancy, interpersonal stress increases within romantic partnerships, and potential lack of involvement from the fatherof-the-child is related to higher levels of depression symptoms in adolescents [36, 37]. Inadequate social support, perceived stress, and depression symptoms have been associated with excess GWG and postpartum weight retention [38-41]. In pregnant adolescents, prevalence of elevated depression symptoms (14-48\%) is doubled compared to both non-pregnant adolescents and to adult pregnant women [42-45]. Adolescents who gain weight rapidly and who have greater total GWG report the highest levels of depression during pregnancy, as compared to adolescents who have slow or adequate GWG [46]. As displayed in Fig. 1, we propose that poor social functioning may contribute to perceived stress and depression symptoms, which promote excess GWG through mechanisms such as overeating in an attempt to cope with negative emotions, physical inactivity, and sleep disturbance [47-52].

Therefore, interventions designed for pregnant adolescents to decrease interpersonal conflict, improve social support, and reduce perceived stress and depression offer the potential to serve as a novel approach to mitigate excess GWG. Consistent with this possibility, one cluster-randomized trial compared group prenatal care with usual individual care for improving reproductive outcomes among pregnant adolescents and young women (ages 14-21 years). In secondary analyses, the investigators found that those in group care showed greater reductions in perinatal depression symptoms [53] and had less GWG and less weight retention at 12months postpartum [54]. In a separate study, a retrospective chart review analysis showed that pregnant adolescents who had received group prenatal care were more likely to have met GWG guidelines than adolescents who had received individual care [55]. While group prenatal care offers a promising approach to excess GWG in adolescent pregnancy, there are challenges to centering group care in pregnant teenagers. Group care can be difficult to coordinate in terms of timing of patients' gestational age, appointment cancellations, and less overall flexibility to accommodate individual schedules.

Alternatively, interpersonal interventions to target social-emotional difficulties merit testing to address the problem of excess GWG in adolescent pregnancy by improving social functioning, perceived stress, and depression. Previous studies with adult pregnant women have shown that interpersonal interventions are associated with improvements in depression symptoms. For example, adult pregnant women assigned to a 16-week group-based interpersonal psychotherapy (IPT) program demonstrated greater improvements in depression symptoms as compared to those assigned to a parent education program [56, 57]. Moreover, adult pregnant women assigned to a culturally relevant, enhanced brief IPT (i.e., 8 individual sessions during pregnancy and maintenance sessions up to 6-months postpartum) showed improvements in social functioning and depression symptoms at 6-months postpartum, as compared to enhanced usual care [58].

\section{Study objectives}

The current study is a randomized controlled pilot and feasibility study. The primary aim is to determine the feasibility and acceptability of a 6-session adolescent IPT program for prevention of excess GWG. We selected IPT based on its demonstrated effectiveness in reducing depression symptoms and improving social functioning in non-pregnant adolescents, including adolescents from diverse racial/ethnic groups and socioeconomic backgrounds, as well as pregnant adults [56-61]. Moreover, IPT has demonstrated effectiveness in non-pregnant adolescents in targeting other posited mechanisms for excess weight gain, such as emotional eating and bingeeating [62]. IPT is delivered as a relatively brief, manualized program using individual sessions every $2-3$ weeks, throughout 12- to 30-weeks gestation. We are assessing feasibility and acceptability of IPT as delivered within the setting of an interdisciplinary adolescent pregnancy hospital clinic. The secondary aim is to estimate the effect of IPT integrated into usual prenatal care, in comparison to usual care only, on key healthcare outcomes grounded in the theoretical framework, including perinatal social functioning, perceived stress, depression symptoms, eating behavior, physical activity, sleep, GWG, delivery outcomes, and at 3-months postpartum, 


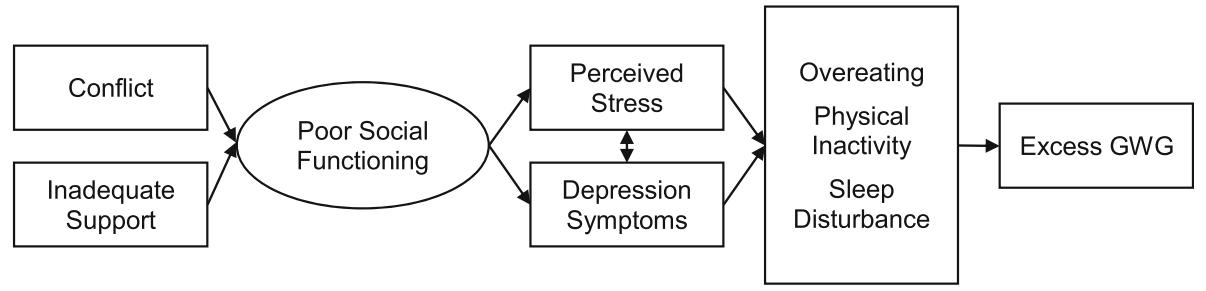

Fig. 1 Theoretical model of interpersonal problems and excess gestational weight gain (GWG)

maternal weight retention, maternal/infant adiposity, and maternal insulin sensitivity.

\section{Methods/design}

\section{Setting, inclusion criteria, and recruitment}

Enrollment for the current study takes place at the Colorado Adolescent Maternity Program (CAMP) clinic at Children's Hospital Colorado, University of Colorado School of Medicine/Anschutz Medical Campus in Aurora, Colorado. The CAMP clinic is an interdisciplinary adolescent perinatal program that includes an obstetrician/gynecologist, a nurse midwife, nurse practitioners, dieticians, social workers, psychologists, and case coordinators.

Inclusion criteria for the study are: [1] age 13-19 years [2]; pregnant, 12-18 weeks gestation; and [3] patient receiving care at the CAMP clinic. Exclusion criteria are [1] current full-syndrome psychiatric disorder that, in the opinion of the study investigators, would impede study compliance and/or necessitate more intensive treatment, such as conduct disorder, schizophrenia, or major depressive disorder with active suicidal ideation [2]; regular medication use likely to affect mood or weight, such as anti-depressants or stimulants [3]; highrisk pregnancy complications, such as preeclampsia, gestational diabetes, hypertension, multiple gestation, placenta previa, membrane rupture, or incompetent cervix [4]; major renal, hepatic, or endocrinological disorder, such as hyperthyroidism or Cushing syndrome, or a pulmonary disorder other than mild asthma; and [5] prepregnancy $\mathrm{BMI}<5$ th percentile for age/sex.

The main recruitment method involves presenting the study in-person during prenatal intake visits to adolescents who appear to be eligible based upon review of the hospital electronic medical record. Trained research staff review the medical record as an initial screen of inclusion/exclusion criteria, including a review of adolescent age, gestational age, known pregnancy complications, medication use, and any major medical disorders that would be exclusionary. Adolescents who appear to be eligible based upon the medical record review are visited at an initial prenatal appointment by trained research staff, including nurses from the Perinatal Clinical and Translational Research Center (CTRC) at Children's
Hospital Colorado, who briefly describe the study, assess interest, and give patients a flyer and consent form to review. Participants who are interested after this initial visit are contacted by a study team member to schedule an initial screening visit at the clinic. Other recruitment methods include posting flyers in the clinic and mailing letters and flyers to CAMP patients.

\section{Consent}

Research staff trained to work in research involving human subjects obtain written and active informed consent from potential research participants in a quiet, private room in the CAMP clinic at the screening/baseline study visit. Pregnant minors are able to consent themselves because, according to Colorado State Law, a pregnant minor is emancipated to approve prenatal, delivery, and post-delivery medical care for herself related to the intended live birth of a child (Colorado Revised Statute 13-22-103.5). Patients seeking services in the CAMP clinic typically attend appointments without a parent or guardian present; thus, requiring parental consent for participation would potentially exclude participants and bias findings. Additionally, study participation involves minimal risk. To participate in the 3months postpartum maternal adiposity/insulin sensitivity assessments, participants must either be $\geq 18$ years to provide informed consent or have a parent/guardian provide consent.

\section{Randomization and interventions}

Adolescents who are determined to be eligible upon completion of the baseline assessment are randomized to IPT + usual CAMP clinic care or to usual care only. Randomization is stratified by age (13-16 years versus 17-19 years), weight status (normal weight, BMI 5-84th percentile versus overweight/obesity, BMI $\geq 85$ th percentile), race (Non-Hispanic White versus Other Race/ Ethnicity), and baseline depression symptom level (Center for Epidemiological Studies-Depression Scale (CESD) survey total score $<21$ versus CES-D total score $\geq$ 21). Randomization strings were generated by an electronic program with permuted blocks. 


\section{CAMP usual care}

Participants in both conditions continue to receive adolescent-focused interdisciplinary prenatal care at the CAMP clinic. Adolescent-focused prenatal care generally differs from adult-focused prenatal care by providing developmentally appropriate services that are tailored to meet the unique social-emotional needs of adolescents [63]. More specifically, adolescent-focused prenatal care addresses co-occurring psychosocial stressors that are more prevalent among pregnant adolescents as compared to pregnant adults, such as mental health problems, substance abuse and use, school problems, family conflict, and poverty [35]. At the CAMP clinic, in addition to usual obstetrical prenatal care, CAMP patients are seen a minimum of 4 times by clinic social workers, once for a psychosocial intake and 3 additional times during pregnancy. Additional social worker sessions are scheduled as needed. These meetings provide information about mood changes in pregnancy and basic education on postpartum depression. All patients also meet once with a dietician during the first trimester to review healthy weight gain and nutrition in pregnancy. Additional dietician appointments are scheduled if concerns about inadequate weight gain emerge or in the event of significant nausea and/or vomiting.

\section{Interpersonal psychotherapy (IPT)}

Adolescents randomized to IPT + usual care also are scheduled for the IPT program, "Healthy Relationships, Healthy Weight." This program was adapted from the IPT for the Prevention of Excess Weight Gain (IPTWG) manual, which was designed for use in nonpregnant adolescents at risk for excess weight gain because of above-average weight and disordered eating/ loss-of-control eating patterns [62]. The IPT-WG program is a 12-session group intervention based on the interpersonal theoretical model, which posits that conflictual interactions and lack of social support can worsen or maintain negative affect [64]. Negative affect, in turn, can lead to overeating when not hungry or overeating highly palatable, energy-dense foods (e.g., sweets and snacks), which taken together, lead to excess weight gain [64].

The adaptation of IPT-WG for the current study includes 3 key modifications. First, sessions are delivered in an individual versus weekly group format to facilitate flexible scheduling. Facilitators make every effort to schedule in-person sessions so that they occur immediately before or after usual prenatal care appointments at the CAMP clinic to minimize participant burden. Depending on participant preference, facilitators are available to schedule sessions at the CAMP clinic during normal business hours, independent of usual prenatal care. Moreover, sessions are scheduled once every 2-3 weeks starting after the baseline and screening visit and ending by $28-30$ weeks gestation, thus providing support to adolescents throughout their pregnancy. We chose not to offer telephone sessions in this pilot feasibility study in order to explicitly evaluate feasibility and acceptability of delivering an in-person program within an interdisciplinary adolescent perinatal healthcare setting. Second, the number and duration of sessions is decreased from 12 90-minute sessions to 6 60-minute sessions. This change was based upon preliminary survey data from CAMP clinic patients that indicated a preference for 6 or fewer sessions. Finally, session content is specifically tailored to address typical issues associated with adolescent pregnancy.

A summary of session content for this adaptation of IPT for the prevention of excess GWG is provided in Table 1. The first session focuses on psychoeducation about the connections among social relationships, mood, eating behavior, and healthy weight gain in pregnancy. This initial session also includes a brief interpersonal inventory to assess adolescents' current relationships and to formulate explicit program goals centered on addressing conflict and support in current relationships. The second session focuses on communication analysis, especially nonverbal aspects of communication. The third, fourth, and fifth sessions involve learning new communication skills and applying these communication skills to current relationships with an aim to increase support or reduce conflict. Finally, the sixth session involves planning for using new communication skills in the context of the transition of delivery and psychoeducation about help-seeking in the future. Participants are encouraged to apply skills learned in each session to current relationships in between sessions through home practice exercises. These home practice exercises are facilitated by a binder with handouts. Facilitators call and/or text message participants in between sessions to check-in and remind them to complete home practice and attend the following session.

IPT sessions are facilitated by a clinical psychologist or by a graduate student in clinical/counseling psychology, marriage and family therapy, or related field. All intervention facilitators are trained by a program developer (LBS) in the administration of the IPT program and receive clinical supervision on audio-recorded sessions from a program developer and licensed clinical psychologist.

At the beginning of each session, participants' height and weight are measured and participants also report their mood on a Mood Monitoring Questionnaire. Any participant whose mood worsens considerably or who develops a psychiatric issue necessitating additional treatment is immediately referred. In the event of an acute psychiatric crisis (e.g., active suicidal ideation), the 
Table 1 Summary of IPT session content for the randomized controlled pilot study protocol

\begin{tabular}{|c|c|}
\hline Session & Content \\
\hline 1 & $\begin{array}{l}\text { Introduction; psychoeducation about weight gain in pregnancy; theoretical model of social relationships, mood, and eating patterns; } \\
\text { interpersonal inventory; identify program goals; assign daily journal }\end{array}$ \\
\hline 2 & Affective expression, communication analysis \\
\hline 3 & $\begin{array}{l}\text { New communication skills: "Strike while the iron is cold," "Using 'I' statements," and "Be specific." Script and role-play a conversation with } \\
\text { new skills to be assigned as home practice before next session }\end{array}$ \\
\hline 4 & $\begin{array}{l}\text { New communication skills: "Put yourself in their shoes" and "What you don't say speaks volumes." Script and role-play a conversation } \\
\text { with skills to be assigned as home practice before next session }\end{array}$ \\
\hline 5 & $\begin{array}{l}\text { New communication skills: "Have a few solutions in mind" and "Don't give up." Script and role-play a conversation with skills to be } \\
\text { assigned as home practice before next session }\end{array}$ \\
\hline 6 & Program review; planning ahead for transition of delivery and caring for baby; graduation \\
\hline
\end{tabular}

on-call child psychiatrist at Children's Hospital Colorado is paged for an immediate consult, evaluation, and possible referral to the Emergency Department.

\section{Outcomes}

Primary and secondary outcomes are summarized in Table 2.

\section{Primary outcomes}

Feasibility of study Feasibility will be defined according to several metrics, including feasible recruitment, enrollment, and retention. Estimates are derived from previous longitudinal studies with pregnant women [65, 87-90]. Feasible recruitment will be defined as identifying at least 480 potentially eligible patients based upon medical record review by research staff to approach during the prenatal intake visit. Feasible enrollment will be defined as approximately 25\% (120/480) of preliminary eligible patients agreeing to schedule a screening/baseline assessment and about 67\% (80/120) attending the assessment and enrolling in the study. Feasible enrollment will be estimated as $60 \%(50 / 80)$ of enrolled patients being eligible to be randomized. Finally, feasible retention will be assessed as at least 75\% (38/50) of randomized participants successfully completing at least three of four assessment intervals, including the baseline/screening assessment. This definition of feasible retention refers to the assessment intervals only and does not include the IPT sessions.

Acceptability of IPT Acceptability will first be measured by IPT session attendance. Acceptable IPT session attendance will be defined by $50 \%$ of participants randomized to IPT attending 5 or $6(\geq 80 \%)$ of the total 6 sessions [87-90]. Acceptability will also be measured by participant ratings on a program acceptability interview adapted for the current study from the Treatment Process Questionnaire and administered by a project staff at the end of treatment assessment [66]. This questionnaire asks participants to report on their reasons for enrolling in the study, what they liked and did not like about the IPT program and the perceived impact of the IPT program on their mood and health. Acceptable participant ratings will be defined as above-average ratings.

\section{Secondary outcomes}

Social functioning, perceived stress, and depression Participants complete self-report questionnaires assessing social functioning, perceived stress, and depression on a computer through Research Electronic Data Capture (REDCap). A project staff member reviews completed questionnaires for missing items before the conclusion of the study visit and invites participants to respond to those missing items.

Social functioning is measured by two questionnaires. The Social Adjustment Scale-Self-Report (SAS-SR) is a 23-item questionnaire assessing social role functioning in several domains including peer, family, romantic relationships, and school/work [67]. Higher scores indicate worse social functioning. The Network of Relationships Inventory-Behavioral Systems Version (NRI-BSV) is a 28-item questionnaire assessing five social support features and three negative interaction features [68]. Stress is assessed as a continuous measure of stress perception by the Perceived Stress Scale (PSS), a 10-item questionnaire [69]. Higher scores suggest greater stress perception. Depression is measured by two questionnaires. The Center for Epidemiological Studies-Depression Scale (CES-D), a 20-item questionnaire assessing depression as a continuous measure of symptoms [70]. Depression is also assessed as a continuous measure of symptoms on the Edinburgh Postnatal Depression Scale (EPDS), a 10 -item questionnaire [71, 72]. Higher scores on the CES-D and the EPDS are indicative of more elevated depression symptoms. Finally, depression diagnosis is assessed via the Mini-International Neuropsychiatric Interview for Children and Adolescents (MINI-KID), a structured diagnostic interview for psychiatric disorders in children and adolescents. 
Table 2 Overview of constructs and assessments throughout the randomized controlled pilot study protocol

\begin{tabular}{|c|c|c|c|c|}
\hline Construct & Measurement & Description & Intervals assessed & Reference \\
\hline \multicolumn{5}{|l|}{ Primary outcomes } \\
\hline Feasibility of study & & $\begin{array}{l}\text { Number of eligible participants, randomized participants, } \\
\text { retention and attrition of randomized participants }\end{array}$ & $\begin{array}{l}\text { Baseline, mid-pregnancy, post- } \\
\text { program, 3-month postpartum }\end{array}$ & [65] \\
\hline Acceptability of IPT & & $\begin{array}{l}\text { IPT session attendance ( } \geq 80 \% \text { ), above-average participant } \\
\text { IPT program ratings }\end{array}$ & Post-program & [66] \\
\hline \multicolumn{5}{|l|}{ Secondary Outcomes } \\
\hline \multirow[t]{2}{*}{ Social functioning } & SAS-SR & $\begin{array}{l}\text { 24-item self-report scale of interpersonal functioning in family, } \\
\text { friend, romantic, and school or work domains }\end{array}$ & $\begin{array}{l}\text { Baseline, mid-pregnancy, post- } \\
\text { program, 3-month postpartum }\end{array}$ & [67] \\
\hline & NRI-BSV & $\begin{array}{l}\text { 28-item self-report scale of relationship characteristics for } \\
\text { mother, father, peer, and romantic partner relationships }\end{array}$ & & [68] \\
\hline Perceived stress & PSS & 14-item self-report scale to assess perception of stress & $\begin{array}{l}\text { Baseline, mid-pregnancy, post- } \\
\text { program, 3-month postpartum }\end{array}$ & [69] \\
\hline \multirow[t]{3}{*}{ Depression } & CES-D & 20-item self-report scale to assess depression symptoms & $\begin{array}{l}\text { Baseline, mid-pregnancy, post- } \\
\text { program, 3-month postpartum }\end{array}$ & [70] \\
\hline & EPDS & 10-item self-report scale to assess depression symptoms & $\begin{array}{l}\text { Baseline, mid-pregnancy, post- } \\
\text { program, 3-month postpartum }\end{array}$ & {$[71,72]$} \\
\hline & MINI-KID & $\begin{array}{l}\text { Structured clinical interview to assess psychiatric disorders, } \\
\text { such as major depressive disorder }\end{array}$ & $\begin{array}{l}\text { Baseline, post-program, 3-month } \\
\text { postpartum }\end{array}$ & [73] \\
\hline \multirow[t]{2}{*}{ Disinhibited eating } & EDE & $\begin{array}{l}\text { Semi-structured interview to assess disordered eating } \\
\text { including objective binge, subjective binge, and objective } \\
\text { overeating }\end{array}$ & $\begin{array}{l}\text { Baseline, post-program, 3-month } \\
\text { postpartum }\end{array}$ & {$[74,75]$} \\
\hline & EES-C & $\begin{array}{l}25 \text {-item self-report questionnaire to assess eating in } \\
\text { response to negative emotions }\end{array}$ & $\begin{array}{l}\text { Baseline, mid-pregnancy, post- } \\
\text { program, 3-month postpartum }\end{array}$ & [76] \\
\hline Physical activity & $\begin{array}{l}\text { ActiGraph } \\
\text { GT3X+ }\end{array}$ & $\begin{array}{l}\text { Body-worn accelerometer to measure } 7 \text { days and nights } \\
\text { of habitual physical activity including step counts, light } \\
\text { and moderate-vigorous intensity, and sedentary time }\end{array}$ & $\begin{array}{l}\text { Baseline, mid-pregnancy, post- } \\
\text { program }\end{array}$ & {$[77,78]$} \\
\hline Sleep disturbance & $\begin{array}{l}\text { ActiGraph } \\
\text { GT3X+ }\end{array}$ & $\begin{array}{l}\text { Body-worn accelerometer to measure } 7 \text { nights of sleep including } \\
\text { total sleep time, sleep onset latency, wake after sleep } \\
\text { onset, and sleep efficiency }\end{array}$ & $\begin{array}{l}\text { Baseline, mid-pregnancy, post- } \\
\text { program }\end{array}$ & {$[79,80]$} \\
\hline Body composition & BMl indices & Height and weight measured to calculate BMI & $\begin{array}{l}\text { Baseline, mid-pregnancy, post- } \\
\text { program, 3-month postpartum }\end{array}$ & [81] \\
\hline $\begin{array}{l}\text { Maternal postpartum } \\
\text { adiposity }\end{array}$ & Body fat & $\begin{array}{l}\text { DXA conducted to measure body composition including } \\
\text { total fat and lean mass }\end{array}$ & 3-month postpartum & {$[82,83]$} \\
\hline $\begin{array}{l}\text { Maternal insulin } \\
\text { sensitivity }\end{array}$ & $\begin{array}{l}\text { WBISI, QUICKI, } \\
\text { HOMAIR }\end{array}$ & $\begin{array}{l}\text { 7-sample, } 2 \text {-h oral glucose tolerance test to estimate } \\
\text { insulin sensitivity }\end{array}$ & 3-month postpartum & {$[84,85]$} \\
\hline Infant adiposity & Body fat & $\begin{array}{l}\text { Infant PeaPod conducted to measure body composition } \\
\text { including total fat and lean mass }\end{array}$ & 3-month postpartum & {$[86]$} \\
\hline
\end{tabular}

Baseline baseline/screening assessment occurring around 12-18 weeks gestation; Mid-Pregnancy mid-pregnancy assessment occurring around 21-28 weeks gestation; Post-Program post-program assessment occurring around 30-34 weeks gestation; 3 Month Postpartum assessment occurring around postpartum week 12; SAS-SR Social Adjustment Scale, Self-Report; NRI-BSV Network of Relationships Inventory-Behavioral Systems Version; PSS Perceived Stress Scale; CES-D Center for Epidemiologic Studies-Depression Scale; EPDS Edinburgh Postnatal Depression Scale; MINI-KID Mini-International Neuropsychiatric Interview for Children and Adolescents; EDE Eating Disorder Examination; EES-C Emotional Eating Scale-Adapted for Children; BMI body mass index (kg/m², $z$-score, percentile); DXA dualenergy X-ray absorptiometry; WBISI whole body insulin sensitivity index; QUICKI quantitative insulin sensitivity check index; HOMAIR homeostasis model assessment of insulin resistance

Eating behavior The overeating section of the Eating Disorders Examination (EDE) Version 14.0, a semistructured clinical interview [74, 75], is administered by trained research staff to assess overeating episodes with and without a feeling of subjective loss-of-control. The EDE has demonstrated good reliability and validity in adolescent samples [91]. Emotional eating, referring to eating in response to negative emotions, is assessed with the Emotional Eating Scale-Adapted for Children and Adolescents (EES-C) [76]. The EES-C is a 25-item self- report questionnaire that has shown good psychometric properties in adolescent samples [76].

Physical activity and sleep disturbance Habitual physical activity, sedentary behavior, and sleep disturbance are derived from ActiGraph GT3X+ accelerometers (ActiGraph, Pensacola, FL), which have well-established validity and reliability in adolescents [77, 79]. These lightweight monitors are worn for seven days and nights on participants' non-dominant wrist and are only 
removed for water-based activities, such as showering or swimming. Accelerometers record data with a sampling frequency of $30 \mathrm{~Hz}$ using 60 -second epochs. Light physical activity, moderate-to-vigorous activity, and sedentary time are classified based on mean counts per epoch according to established guidelines [78]. Parameters of sleep disturbance include total sleep time, sleep onset latency, wake after sleep onset, and sleep efficiency [80].

Gestational weight gain Weight is derived to the nearest $0.1 \mathrm{~kg}$ on a calibrated digital scale, and height is derived by a stadiometer in triplicate. Weight and height will also be derived from the medical record for intervals that do not coincide with a study assessment interval. More specifically, weight and height will be derived from the medical records at prenatal appointments, at delivery, and at postnatal appointments. Continuous GWG during pregnancy will be measured using two metrics: [1] GWG during the experimental phase, as defined by the difference between measured maternal weight at the baseline assessment to the post-program assessment, and [2] total GWG, as defined by the difference between self-reported prepregnancy weight and maternal weight at delivery, derived from the medical record. Classification of pre-pregnancy weight status as normal weight, overweight, or obese will be based on CDC BMI percentile for age [81]. Categorical GWG, defined as appropriate versus excessive, will be measured relative to Institute of Medicine guidelines for gestational age for both GWG during the experimental phase and total GWG.

Maternal postpartum weight retention Continuous maternal postpartum weight retention will be measured as the difference in self-reported pre-pregnancy weight and measured maternal weight at the 3-month postpartum assessment [39].

Maternal postpartum adiposity For those who participate in the adjunct metabolic assessments, participants have a dual energy X-ray absorptiometry (DXA) scan to evaluate body composition, including total lean mass and total fat mass, using Hologic QDR Discovery A (S/ N81337; Bedford, MA) [82]. DXA has demonstrated good validity in youth [83].

Maternal postpartum insulin sensitivity Maternal insulin sensitivity is derived from a 2-hour oral glucose tolerance test at the 3-month postpartum interval. In the morning following a 10-hour overnight fast, participants ingest $1.75 \mathrm{~g} / \mathrm{kg}$ of glucola ( $\max =75 \mathrm{~g}$ ). Blood is sampled via an intravenous line for insulin, glucose, and c-peptide at fasting, 10, 20, 30, 60, 90, and 120 min after ingesting the glucola [85]. Insulin sensitivity will be estimated with the whole body insulin sensitivity index (WBISI), as well as the quantitative insulin sensitivity check index (QUICKI) and the homeostasis model assessment of insulin resistance (HOMA-IR). These methods of measuring insulin sensitivity have been validated against euglycemichyperinsulinemic clamp-derived measures in youth with overweight and obesity [84].

Infant adiposity At the 3-month postpartum assessment, infants will complete a PEAPOD to evaluate fat mass and fat-free mass using air displacement plethysmography [86].

\section{Participant timeline}

All assessments take place at the CAMP clinic, with the exception of the 3-month postpartum assessment, which takes place at the Outpatient Pediatric CTRC at Children's Hospital Colorado. Participant timeline is presented in Table 3.

\section{Screening/baseline assessment}

Potentially eligible and interested volunteers are invited to participate in a screening and baseline assessment appointment. This appointment takes approximately three hours. A trained research staff member obtains informed written consent. Participants complete online questionnaires via REDCap to assess social functioning, perceived stress, depression symptoms, and eating behaviors. A project staff member administers a structured clinical interview to rule out the presence of a psychiatric disorder, current self-injurious behavior, or current suicidal behavior that would warrant more intensive treatment. Participants who meet criteria for a current fullsyndrome psychiatric disorder or who report current and active suicidal ideation are immediately referred to CAMP behavioral health staff for further evaluation and treatment. At the screening visit, project staff also administer a semi-structured interview to assess eating patterns, including overeating and binge eating, and review a 3-day diet record. Research staff who administer clinical interviews are trained by clinical psychologists through a combination of didactics, role-playing, and live supervision. Clinical interviews are audio-recorded and reviewed by clinical psychologists on the research team for fidelity to the interview protocol. At the end of the screening visit, participants are fitted with an ambulatory accelerometer that they are asked to wear for seven days following the study visit to measure habitual physical activity. Participants are paid $\$ 50$ for completing this screening visit. At the end of the screening visit, eligible participants are randomized to either IPT + usual care or usual care only. 
Table 3 Overview of a participant's timeline, assessment intervals, and core measures

\begin{tabular}{|c|c|c|c|c|c|c|c|c|c|c|c|c|}
\hline \multirow[b]{3}{*}{ Gestational week } & \multicolumn{12}{|l|}{ Study Period } \\
\hline & \multirow[t]{2}{*}{ Initiation of prenatal care } & \multirow{2}{*}{$\begin{array}{l}\text { Allocation } \\
12-18\end{array}$} & \multicolumn{6}{|c|}{ Intervention } & \multicolumn{3}{|c|}{ Post-intervention } & \multirow[t]{2}{*}{ 3-month post-partum } \\
\hline & & & 24 & 26 & 28 & 30 & 32 & 34 & 36 & 38 & 40 & \\
\hline \multicolumn{13}{|l|}{ Enrollment } \\
\hline Eligibility Screen & $x$ & & & & & & & & & & & \\
\hline Informed Consent & & $x$ & & & & & & & & & & \\
\hline Allocation & & $x$ & & & & & & & & & & \\
\hline \multicolumn{13}{|l|}{ Interventions } \\
\hline IPT & & & $x$ & $x$ & $x$ & $x$ & $x$ & $x$ & & & & \\
\hline \multicolumn{13}{|l|}{ UC } \\
\hline Medical & $x$ & $x$ & $x$ & $x$ & $x$ & $x$ & $x$ & $x$ & $x$ & $x$ & $x$ & $x$ \\
\hline Nutrition & $x$ & & & $x$ & & & & & & & & \\
\hline Social work & $x$ & & & $x$ & & & & & $x$ & & & \\
\hline \multicolumn{13}{|l|}{ Assessments } \\
\hline Feasibility & $x$ & $x$ & $x$ & $x$ & $x$ & $x$ & $x$ & $x$ & $x$ & & & $x$ \\
\hline Acceptability & $x$ & $x$ & $x$ & $x$ & $x$ & $x$ & $x$ & $x$ & $x$ & & & \\
\hline Social functioning & & $x$ & & & $x$ & & & & $x$ & & & $x$ \\
\hline Perceived stress & & $x$ & & & $x$ & & & & $x$ & & & $x$ \\
\hline Depression & $x$ & $x$ & $x$ & $x$ & $x$ & $x$ & $x$ & $x$ & $x$ & & & $x$ \\
\hline Eating behavior & & $x$ & & & $x$ & & & & $x$ & & & $x$ \\
\hline Physical activity & & $x$ & & & $x$ & & & & $x$ & & & \\
\hline Sleep disturbance & & $x$ & & & $x$ & & & & $x$ & & & \\
\hline Height/weight & $x$ & $x$ & $x$ & $x$ & $x$ & $x$ & $x$ & $x$ & $x$ & $x$ & & $x$ \\
\hline Maternal insulin sensitivity & & & & & & & & & & & & $x$ \\
\hline Maternal adiposity & & & & & & & & & & & & $x$ \\
\hline Infant adiposity & & & & & & & & & & & & $x$ \\
\hline
\end{tabular}

IPT interpersonal psychotherapy, UC usual care

\section{Mid-program assessment}

Participants complete a brief assessment, estimated to take no longer than $30 \mathrm{~min}$, halfway through the experimental phase, corresponding to about 6-9 weeks after the baseline assessment. Participants repeat questionnaires assessing social functioning, stress, depression symptoms, and eating behaviors. They also re-wear the accelerometer for seven days following the study visit to re-assess habitual physical activity. Participants receive a pack of newborn diapers for completing this midprogram assessment.

\section{Post-program assessment}

Participants complete an assessment at the end of the experimental phase, which corresponds to about 12-18 weeks after the baseline assessment and just prior to delivery. This assessment takes about two hours. Participants repeat the same research tasks as at the baseline assessment. In addition, a project staff member administers a program acceptability interview to adolescents who were randomized to the IPT condition. Participants are paid $\$ 75$ for completing the post-program assessment.

\section{3-month postpartum assessment}

Participants complete a final assessment at 3-months postpartum, a time when inter-individual differences in postpartum weight retention and postpartum depression emerge [92]. Participants repeat the same research tasks at the baseline assessment. Participants' 3-month old babies complete an infant body composition assessment using air displacement plethysmography (PEAPOD). A project staff member reviews the medical record to assess measured height and weight at delivery and postpartum appointments, as well as any pregnancy or birth complications. Participants are paid $\$ 75$ for completing the 3-month postpartum assessment.

Participants are invited to opt in, or out, of an additional adjunct metabolic study, which includes a maternal DXA scan of post-partum adiposity and a 2-h oral glucose tolerance test (OGTT) to estimate maternal 
post-partum insulin sensitivity. Participants are paid $\$ 75$ for the adjunct metabolic procedures.

\section{Sample size}

We plan to randomize 50 out of $80(60 \%)$ enrolled participants following a screening/baseline eligibility assessment. Our estimation of a total sample size of $N=50$ (i.e., $n=25$ per condition) is based on recommendations for a two-arm superiority pilot trail in which small-tomoderate standardized effects are anticipated [93]. Furthermore, our sample size estimation is consistent with recommendations for pilot randomized trials using an $80 \%$ one-sided confidence interval approach to exclude a clinically important difference between study arms [94].

\section{Statistical analysis}

Baseline participant characteristics and feasibility/acceptability findings will primarily be measured with descriptive statistics, including mean with standard deviation, median with interquartile range, frequency, and percentage. Analysis of covariance (ANCOVA) will be used to evaluate group differences (IPT + usual care versus usual care) in secondary outcomes, including social functioning, perceived stress, depression symptoms, eating behaviors, physical activity, sleep disturbance, maternal post-partum insulin sensitivity, maternal post-partum adiposity, and infant adiposity. We will use 95\% confidence intervals to measure precision of the estimated differences between conditions, per recommendations [95]. Given that this is a pilot study, results will be considered preliminary. Although our theoretical framework suggests that IPT + usual care, as compared to usual care only, will impact these secondary outcomes via a series of mechanistic pathways, this pilot feasibility study is not sufficiently powered to test mediation. Covariates considered in these models will include baseline maternal age, gestational age, race/ethnicity, baseline CES-D score, and pre-pregnancy BMI/weight status. Missing data will be examined for patterns of missingness and imputation, with the intent-to-treat sample, will be used if deemed appropriate. Given the pilot nature of the study, we also will conduct sensitivity analyses using listwise deletion with complete data.

\section{Ethics and dissemination}

The protocol has been approved by a single Institutional Review Board, the Colorado Multiple Institutional Review Board (COMIRB), which provides oversight for the University of Colorado School Of Medicine and Children's Hospital Colorado. An Institutional Review Board authorization agreement was established between the Institutional Review Board of Colorado State University, an administrative site, and COMIRB, in order for COMIRB to serve as the primary IRB. We follow
Institutional Review Board requirements pertaining to reporting of unanticipated problems and adverse events.

Personal information is collected directly from research participants and from their medical record. Personal information is stored separately from other research data and will not be shared with anyone outside of the research team, which includes personnel at the University of Colorado, Children's Hospital Colorado, and Colorado State University, with the exception of entities that monitor human subject research, such as COMIRB. Electronic data are stored and managed by REDCap and a secure server at the University of Colorado. The REDCap system is password protected and only accessible by research staff members. Digital audio recordings from interviews and interventions are uploaded to password-protected folders on a secure server at the University of Colorado. After uploading, recordings are deleted from the digital recording device. Paper data are stored in locked cabinets in administrative research space at Children's Hospital Colorado. All data will be preserved for seven years following IRB acknowledgement of study closure. Results and conclusions from the current study will be disseminated by publication in peer-reviewed journals and conference presentations. We also will disseminate findings to clinic providers who have been involved in supporting the study.

\section{Discussion}

The current study is a randomized controlled feasibility study piloting a novel approach to the prevention of excess GWG in adolescent pregnancy. The primary aim is to assess the feasibility and acceptability of a relatively brief, 6-session individual IPT program, delivered within the context of an interdisciplinary adolescent pregnancy hospital clinic. The secondary aim is to explore whether there are health benefits to pregnant adolescents who receive IPT-in perinatal social functioning, perceived stress, depression symptoms, eating behaviors, physical activity, sleep, GWG, and postpartum maternal and infant metabolic health-as compared to the usual care that pregnant adolescents would receive in an interdisciplinary adolescent maternity clinic.

Preventative interventions for excess GWG in adult women have previously focused on lifestyle modification, including healthy eating and physical activity. The available literature suggests that lifestyle-based interventions require significant financial and time resources, and unfortunately, generally demonstrate insufficient effectiveness [29]. Moreover, no study to date has been specifically designed to evaluate a prevention of GWG intervention in pregnant adolescents, even though excess GWG is prevalent in this population and is associated with serious, negative health outcomes [3]. Pregnant 
adolescents face unique socioemotional needs, often including mental health problems, academic problems, family conflict, and poverty [35]. There is growing evidence that such social-emotional factors may interfere with a healthy weight gain in pregnancy through their influence on overeating to cope with negative emotions and stress, reduced physical activity, and disturbed sleep $[96,97]$. From an interpersonal theoretical framework, receipt of an IPT intervention is posited to reduce conflict and increase support in adolescents' interpersonal relationships, which in turn, is theorized to improve perceived stress and negative affect, and ultimately facilitate healthy eating, physical activity, and sleep to influence a healthy GWG.

The proposed pilot randomized feasibility study is the first necessary step in understanding the potential utility of an evidence-based psychotherapy intervention-addressing social functioning, stress, and negative emotions-for prevention of excess GWG in adolescent pregnancy. We anticipate that IPT will be feasible and acceptable to pregnant adolescents. Moreover, we have preliminary data that suggest it will be feasible to incorporate a psychosocial intervention into an interdisciplinary hospital clinic, as evidenced by a $72 \%$ attendance rate in an existing parenting skills training intervention in the CAMP clinic. However, it is possible that there could be obstacles to attendance (e.g., transportation). Some we have anticipated (e.g., providing taxis and bus passes as needed), but others (e.g., school/work schedules) could interfere with sessions. A strength of the individualized nature of our IPT program is that it permits a high degree of flexibility in rescheduling sessions at alternative times.

With respect to secondary outcomes, we anticipate that adolescents who receive IPT, relative to usual care only, will show patterns of improved perinatal social functioning, lower perceived stress, reduced depression symptoms, healthier eating patterns, increased physical activity, improved sleep, less excess GWG, and better postpartum maternal and infant metabolic health. However, IPT may improve social-emotional adjustment without preventing excess GWG or postpartum weight retention and metabolic outcomes. In this case, we would have initial evidence for a brief, potentially costeffective approach to improving social-emotional health in pregnant adolescents, which has important public health relevance for postpartum depression prevention and maternal-infant health, given the serious, negative effects of depression on maternal-infant psychosocial outcomes [98]. Ultimately, results and conclusions have the potential to benefit not only emotional and physical health outcomes for adolescent mothers, but also health outcomes for offspring as part of a larger public health perspective on the intergenerational transmission of depression, obesity, and cardiometabolic disease.
Feasibility and acceptability findings from the current pilot study will inform the development of a larger randomized controlled trial that is adequately powered to measure the effectiveness of this brief individual psychosocial intervention to prevent excess GWG in adolescents. Testing the effectiveness of this psychosocial intervention within the structure of an existing interdisciplinary adolescent maternity hospital clinic is ideal to facilitate successful transfer of research-based knowledge to routine clinical practice. The potential for dissemination of this psychosocial intervention is further enhanced by evidence showing that IPT can be delivered effectively and with fidelity by trained facilitators who do not necessarily have an advanced degree in psychology, such as lay community health workers [99].

We considered the selection of the control group extensively. A limitation of our usual care control is that we will not be able to account for potential confounding effects of attention. Because adolescent patients receive education about depression as part of prenatal usual care, and because we seek to first establish feasibility and acceptability, we felt that comparison to usual care was the optimal, initial step. Depending upon the results, a subsequent well-powered trial might include a 3-group design comparing [1] IPT to [2] usual care and either (3a) an attention-matched educational control or (3b) a standard behavioral lifestyle intervention. Due to the pilot nature of this study, one shortcoming is that we will only have maternal insulin sensitivity and maternal/ infant adiposity at postpartum, and thus, will not be able to control for earlier levels of these variables. These measures are secondary outcomes, and if positive signals are detected from these pilot data, future studies might consider use of repeated measures of maternal metabolic characteristics and measures at birth of infant adiposity. Another limitation of this pilot study is that outcome assessors are not blind to intervention assignment, and thus, assessment of some outcomes may be influenced by expectancy biases (i.e., such as those measured by clinical interview). That being said, a potential strength of the current study is heterogeneity of outcome variables, either by participant self-report or objective measures, such as accelerometers to assess habitual physical activity and sleep. A subsequent randomized controlled effectiveness trial would have the resources to ensure that research study staff who collect outcome data are blind to intervention assignment.

The concept of focusing on pregnant adolescents will allow us to target an underrepresented, underserved group who are likely to face heightened social-emotional challenges as compared to pregnant adult women [35]. Opportunities to intervene with this at-risk group can be expected to help prevent many of these young women, and their future infants, from a lifetime trajectory of 
obesity with its multiple adverse health consequences. The proposed IPT intervention represents a novel approach to addressing excess GWG in adolescents by targeting psychosocial risk factors including social functioning, perceived stress, and depression symptoms.

\section{Abbreviations}

ANCOVA: Analysis of covariance; CAMP: Colorado Adolescent Maternity Program; CES-D: Center for Epidemiological Studies-Depression Scale; COMIRB: Colorado Multiple Institutional Review Board; CTRC: Clinical and Translational Research Center; DXA: Dual energy X-ray absorptiometry; EDE: Eating disorders examination; EES-C: Emotional Eating Scale for Children; EPDS: Edinburgh Postnatal Depression Scale; GWG: Gestational weight gain; HOMA-IR: Homeostasis model assessment of insulin resistance: IPT: Interpersonal psychotherapy; MINI-KID: Mini-International Neuropsychiatric Interview for Children and Adolescents; NRI-BSV: Network of Relationships Inventory-Behavioral Systems Version; OGTT: Oral glucose tolerance test; PSS: Perceived Stress Scale; QUICKI: Quantitative insulin sensitivity check index; REDCap: Research Electronic Data Capture; SASSR: Social Adjustment Scale-Self Report; WBISI: Whole body insulin sensitivity index

\section{Acknowledgements}

The authors extend their gratitude to the multi-disciplinary clinic providers for their support of this study and to the clinic patients who have volunteered to take part. The authors also would like to thank Sona Dimidjian, PhD; Elizabeth Goodman, MD; and Jenn Leiferman, PhD for their various contributions to the early phases of this work.

\section{Authors' contributions}

LBS, KJN, LAB, SMS, and JLS intellectually designed the study. LBS obtained funding for the project. LBS and MT drafted the interpersonal psychotherapy intervention manual for pregnant adolescents, and LDG and BP contributed to adapting the manual. $L B S, L D G$, and BP have delivered the intervention. $L D G, E C, A H$, and BP have coordinated the study and determined protocol modifications to increase the likelihood of feasibility and acceptability. LBS and LDG wrote the manuscript. EC, AH, BP, MT, KJN, LAB, SMS, JLS, LBS, and LDG read and approved the final manuscript.

\section{Funding}

Support for this study was provided by the mini-grant program of the College of Health and Human Sciences at Colorado State University, a faculty seed grant from the Colorado School of Public Health, an interdisciplinary pilot grant from the Prevention Research Center at Colorado State University, and microgrant funding from the Colorado Clinical and Translational Sciences Institute (supported by NIH/NCATS Colorado CTSA grant UL1 TR002535). Contents are the authors' sole responsibility and do not necessarily represent official $\mathrm{NIH}$ views. Funders did not and will not direct the design of the study, data collection, analysis, or interpretation of data.

\section{Availability of data and materials}

The de-identified dataset generated from this protocol will be available from the corresponding author upon reasonable request.

\section{Ethics approval and consent to participate}

An institutional agreement was established between the Colorado State University Institutional Review Board and the Colorado Multiple Institutional Review Board, with the latter as primary. All procedures were approved by the Colorado Multiple Institutional Review Board. The Research Institute of the Children's Hospital Colorado and the Scientific Advisory Research Committee of the Colorado Clinical and Translational Sciences Institute also reviewed and approved the study protocol.

\section{Consent for publication}

Not applicable; no individual or identifying information is published.

\section{Competing interests}

The authors declare that they have no competing interests.

\section{Author details}

'Department of Human Development and Family Studies, Colorado State University, 1570 Campus Delivery, Fort Collins, CO 80523-1570, USA. ${ }^{2}$ Department of Community and Behavioral Health, Colorado School of Public Health, Aurora, CO, USA. ${ }^{3}$ Department of Pediatrics, University of Colorado School of Medicine and Children's Hospital Colorado, Aurora, CO, USA. ${ }^{4}$ Department of Medical and Clinical Psychology and Department of Medicine, Uniformed Services University of the Health Sciences, Department of Defense, Bethesda, MD, USA. ${ }^{5}$ Department of Medicine, University of Colorado School of Medicine, Aurora, CO, USA. ${ }^{6}$ Department of Obstetrics and Gynecology, University of Colorado School of Medicine, Aurora, CO, USA.

Received: 31 March 2019 Accepted: 26 February 2020

Published online: 20 March 2020

\section{References}

1. Martin JA, Hamilton BE, Osterman MJK, Driscoll AK, Drake P. Births: final data for 2017. Natl Vital Stat Rep. 2018;67(8):1-50.

2. Elchert J, Beaudrot M, DeFranco E. Gestational weight gain in adolescent compared with adult pregnancies: an age-specific body mass index approach. J Pediatr. 2015;167(3):579-85.e2.

3. Zamora-Kapoor A, Walker LR. Excessive gestational weight gain in adolescent and adult pregnancies: an overlooked public health problem. J Pediatr. 2015:167(3):515-7.

4. Haeri S, Guichard I, Baker AM, Saddlemire S, Boggess KA. The effect of teenage maternal obesity on perinatal outcomes. Obstet Gynecol. 2009: 113(2):300-4.

5. Aliyu MH, Luke S, Kristensen S, Alio AP, Salihu HM. Joint effect of obesity and teenage pregnancy on the risk of preeclampsia: a population-based study. J Adolesc Health. 2010;46(1):77-82.

6. Sukalich S, Mingione MJ, Glantz JC. Obstetric outcomes in overweight and obese adolescents. Am J Obstet Gynecol. 2006;195(3):851-5.

7. Harper LM, Chang JJ, Macones GA. Adolescent pregnancy and gestational weight gain: do the Institute of Medicine recommendations apply? Am J Obstet Gynecol. 2011;205(2):140.

8. MacSween K, Whelan E, Woolcott CG. Gestational weight gain and perinatal outcomes in adolescent mothers: a retrospective cohort study. J Obstet Gynaecol Can. 2016;38(4):338-45.

9. Groth SW. The long-term impact of adolescent gestational weight gain. Res Nurs Health. 2008;31(2):108-18.

10. Groth SW, Holland ML, Kitzman H, Meng Y. Gestational weight gain of pregnant African American adolescents affects body mass index 18 years later. J Obstet Gynecol Neonatal Nurs. 2013;42(5):541-50.

11. Stevens-Simon C, MCAnarney ER. Adolescent pregnancy: gestational weight gain and maternal and infant outcomes. Am J Dis Child. 1992;146(11): 1359-64.

12. Segel JS, MCAnarney ER. Adolescent pregnancy and subsequent obesity in African-American girls. J Adolesc Health. 1994;15(6):491-4.

13. Hediger ML, Scholl TO, Schall II. Implications of the Camden Study of adolescent pregnancy: interactions among maternal growth, nutritional status, and body composition. Ann N Y Acad Sci. 1997;817:281-91.

14. Mamun AA, Kinarivala M, O'Callaghan MJ, Williams GM, Najman JM, Callaway LK. Associations of excess weight gain during pregnancy with long-term maternal overweight and obesity: evidence from 21 y postpartum follow-up. Am J Clin Nutr. 2010;91(5):1336-41.

15. Whelan E, Armson BA, Ashley-Martin J, MacSween K, Woolcott C. Gestational weight gain and interpregnancy weight change in adolescent mothers. J Pediatr Adolesc Gynecol. 2017;30(3):356-61.

16. Cisneiros RM, Dutra LP, Silveira FJ, Souza AR, Marques M, Amorim MM, et al. Visceral adiposity in the first half of pregnancy predicts newborn weight among adolescent mothers. J Obstet Gynaecol Can. 2013;35(8):704-9.

17. Starling AP, Brinton JT, Glueck DH, Shapiro AL, Harrod CS, Lynch AM, et al. Associations of maternal BMI and gestational weight gain with neonatal adiposity in the Healthy Start study. Am J Clin Nutr. 2015;101(2):302-9.

18. Gunderson EP, Abrams B, Selvin S. The relative importance of gestational gain and maternal characteristics associated with the risk of becoming overweight after pregnancy. Int J Obes Relat Metab Disord. 2000;24(12): 1660-8.

19. Mamun AA, O'Callaghan M, Callaway L, Williams G, Naiman J, Lawlor DA. Associations of gestational weight gain with offspring body mass index and 
blood pressure at 21 years of age: evidence from a birth cohort study. Circulation. 2009;119(13):1720-7.

20. van Rossem L, Wijga AH, Gehring U, Koppelman GH, Smit HA. Maternal gestational and postdelivery weight gain and child weight. Pediatrics. 2015; 136(5):e1294-301.

21. Fraser A, Tilling K, Macdonald-Wallis C, Sattar N, Brion MJ, Benfield L, et al. Association of maternal weight gain in pregnancy with offspring obesity and metabolic and vascular traits in childhood. Circulation. 2010;121(23):2557-64.

22. Barzin M, Hosseinpanah F, Fekri S, Azizi F. Predictive value of body mass index and waist circumference for metabolic syndrome in 6-12-year-olds. Acta Paediatr. 2011;100(5):722-7.

23. Must A, Strauss RS. Risks and consequences of childhood and adolescent obesity. Int J Obes Relat Metab Disord. 1999;23:S2-11.

24. Weiss R, Dziura J, Burgert TS, Tamborlane WW, Taksali SE, Yeckel CW, et al. Obesity and the metabolic syndrome in children and adolescents. N Engl J Med. 2004;350:2362-74.

25. Flegal KM, Kit BK, Orpana H, Graubard BI. Association of all-cause mortality with overweight and obesity using standard body mass index categories: a systematic review and meta-analysis. JAMA. 2013;309(1):71-82.

26. Barbour LA, Hernandez TL. Maternal non-glycemic contributors to fetal growth in obesity and gestational diabetes: spotlight on lipids. Curr Diab Rep. 2018;18(6):37.

27. Dabelea $\mathrm{D}$, Crume T. Maternal environment and the transgenerational cycle of obesity and diabetes. Diabetes. 2011;60(7):1849-55.

28. Gillman MW, Ludwig DS. How early should obesity prevention start? N Engl J Med. 2013;369(23):2173-5.

29. Nicklas JM, Barbour LA. Optimizing weight for maternal and infant health tenable, or too late? Expert Rev Endocrinol Metab. 2015;10(2):227-42.

30. Kamath CC, Vickers KS, Ehrlich A, McGovern L, Johnson J, Singhal V, et al. Behavioral interventions to prevent childhood obesity: a systematic review and metaanalyses of randomized trials. J Clin Endocrinol Metab. 2008;93(12) 4606-15.

31. Klesges RC, Obarzanek E, Kumanyika S, Murray DM, Klesges LM, Relyea GE, et al. The Memphis Girls' health Enrichment Multi-site Studies (GEMS): an evaluation of the efficacy of a 2-year obesity prevention program in African American girls. Arch Pediatr Adolesc Med. 2010;164(11):1007-14.

32. Resnicow K, Yaroch AL, Davis A, Wang DT, Carter S, Slaughter L, et al. GO GIRLS! Results from a nutrition and physical activity program for lowincome, overweight African American adolescent females. Health Educ Behav. 2000:27(5):616-31.

33. Stevens CJ. Obesity prevention interventions for middle school-age children of ethnic minority: a review of the literature. J Spec Pediatr Nurs. 2010;15(3):233-43.

34. Resnicow K, Taylor R, Baskin M, McCarty F. Results of Go Girls: a weight control program for overweight African-American adolescent females. Obes Res. 2005;13(10):1739-48.

35. Hodgkinson S, Beers L, Southammakosane C, Lewin A. Addressing the mental health needs of pregnant and parenting adolescents. Pediatrics. 2014;133(1):114-22.

36. Asselmann E, Wittchen HU, Petzoldt J, Martini J. Peripartum changes in partnership quality among women with and without anxiety and depressive disorders prior to pregnancy: a prospective-longitudinal study. Arch Womens Ment Health. 2016;19(2):281-90.

37. Cabeza de Baca T, Wojcicki JM, Epel ES, Adler NE. Lack of partner impacts newborn health through maternal depression: a pilot study of low-income immigrant Latina women. Midwifery. 2018:64:63-8.

38. Hartley E, McPhie S, Skouteris H, Fuller-Tyszkiewicz M, Hill B. Psychosocial risk factors for excessive gestational weight gain: a systematic review. Women Birth. 2015;28(4):e99-e109.

39. Whitaker K, Young-Hyman D, Vernon M, Wilcox S. Maternal stress predicts postpartum weight retention. Matern Child Health J. 2014;18(9):2209-17.

40. Bodnar LM, Wisner KL, Moses-Kolko E, Sit DK, Hanusa BH. Prepregnancy body mass index, gestational weight gain, and the likelihood of major depressive disorder during pregnancy. J Clin Psychiatry. 2009;70(9):1290-6.

41. Webb JB, Siega-Riz AM, Dole N. Psychosocial determinants of adequacy of gestational weight gain. Obesity (Silver Spring). 2009;17(2):300-9.

42. Barnet B, Joffe A, Duggan AK, Wilson MD, Repke JT. Depressive symptoms, stress, and social support in pregnant and postpartum adolescents. Arch Pediatr Adolesc Med. 1996;150(1):64-9.

43. Deal LW, Holt VL. Young maternal age and depressive symptoms: results from the 1988 National Maternal and Infant Health Survey. Am J Public Health. 1998;88(2):266-70.
44. Kingston D, Heaman M, Fell D, Chalmers B. Maternity experiences study group of the Canadian Perinatal Surveillance System PHAoC. Comparison of adolescent, young adult, and adult women's maternity experiences and practices. Pediatrics. 2012;129(5):e1228-37.

45. Lewinsohn PM, Shankman SA, Gau JM, Klein DN. The prevalence and comorbidity of subthreshold psychiatric conditions. Psychol Med. 2004;34(4): $613-22$.

46. Stevens-Simon C, MCAnarney ER. Determinants of weight gain in pregnant adolescents. J Am Diet Assoc. 1992;92(11):1348-51.

47. Bruch H. Hunger and instinct. J Nerv Ment Dis. 1969;149(2):91-114.

48. Heatherton TF, Baumeister RF. Binge eating as escape from self-awareness. Psychol Bull. 1991;110(1):86-108.

49. Kaplan HI, Kaplan HS. The psychosomatic concept of obesity. J Nerv Ment Dis. 1957;125(2):181-201.

50. Bjorntorp P. Do stress reactions cause abdominal obesity and comorbidities? Obes Rev. 2001;2(2):73-86.

51. Da Costa D, Rippen N, Dritsa M, Ring A. Self-reported leisure-time physical activity during pregnancy and relationship to psychological well-being. J Psychosom Obstet Gynaecol. 2003;24(2):111-9.

52. Downs DS, DiNallo JM, Kirner TL. Determinants of pregnancy and postpartum depression: prospective influences of depressive symptoms, body image satisfaction, and exercise behavior. Ann Behav Med. 2008;36(1): 54-63.

53. Felder JN, Epel E, Lewis JB, Cunningham SD, Tobin JN, Rising SS, et al. Depressive symptoms and gestational length among pregnant adolescents: cluster randomized control trial of CenteringPregnancy(R) plus group prenatal care. J Consult Clin Psychol. 2017;85(6):574-84.

54. Magriples U, Boynton MH, Kershaw TS, Lewis J, Rising SS, Tobin JN, et al. The impact of group prenatal care on pregnancy and postpartum weight trajectories. Am J Obstet Gynecol. 2015;213(5):688.e1-9.

55. Trotman G, Chhatre G, Darolia R, Tefera E, Damle L, Gomez-Lobo V. The effect of centering pregnancy versus traditional prenatal care models on improved adolescent health behaviors in the perinatal period. J Pediatr Adolesc Gynecol. 2015;28(5):395-401.

56. Spinelli MG, Endicott J. Controlled clinical trial of interpersonal psychotherapy versus parenting education program for depressed pregnant women. Am J Psychiatry. 2003;160(3):555-62.

57. Spinelli MG, Endicott J, Goetz RR, Segre LS. Reanalysis of efficacy of interpersonal psychotherapy for antepartum depression versus parenting education program: initial severity of depression as a predictor of treatment outcome. J Clin Psychiatry. 2016;77(4):535-40.

58. Grote NK, Swartz HA, Geibel SL, Zuckoff A, Houck PR, Frank E. A randomized controlled trial of culturally relevant, brief interpersonal psychotherapy for perinatal depression. Psychiatr Serv. 2009;60(3):313-21.

59. Tanofsky-Kraff M, Shomaker LB, Wilfley DE, Young JF, Sbrocco T, Stephens $\mathrm{M}$, et al. Targeted prevention of excess weight gain and eating disorders in high-risk adolescent girls: a randomized controlled trial. Am J Clin Nutr. 2014;100(4):1010-8.

60. Mufson L, Dorta KP, Wickramaratne P, Nomura Y, Olfson M, Weissman MM. A randomized effectiveness trial of interpersonal psychotherapy for depressed adolescents. Arch Gen Psychiatry. 2004;61(6):577-84.

61. Reyes-Portillo JA, McGlinchey EL, Yanes-Lukin PK, Turner JB, Mufson L. Mediators of interpersonal psychotherapy for depressed adolescents on outcomes in Latinos: the role of peer and family interpersonal functioning. Journal of Latina-O Psychology. 2017:5(4):248-60.

62. Tanofsky-Kraff M, Wiffley DE, Young JF, Mufson L, Yanovski SZ, Glasofer DR, et al. A pilot study of interpersonal psychotherapy for preventing excess weight gain in adolescent girls at-risk for obesity. Int I Eat Disord. 2010; 43(8):701-6.

63. Bensussen-Walls W, Saewyc EM. Teen-focused care versus adult-focused care for the high-risk pregnant adolescent: an outcomes evaluation. Public Health Nurs. 2001;18(6):424-35.

64. Tanofsky-Kraff M, Wiffley DE, Young JF, Mufson L, Yanovski SZ, Glasofer DR, et al. Preventing excessive weight gain in adolescents: interpersonal psychotherapy for binge eating. Obesity (Silver Spring). 2007;15(6):1345-55.

65. van Delft K, Schwertner-Tiepelmann N, Thakar R, Sultan AH. Recruitment of pregnant women in research. J Obstet Gynaecol. 2013;33(5):442-6.

66. Hilbert A, Saelens BE, Stein Rl, Mockus DS, Welch RR, Matt GE, et al. Pretreatment and process predictors of outcome in interpersonal and cognitive behavioral psychotherapy for binge eating disorder. J Consult Clin Psychol. 2007;75(4):645-51. 
67. Weissman MM, Bothwell S. Assessment of social adjustment by patient selfreport. Arch Gen Psychiatry. 1976;33(9):1111-5.

68. Furman W, Buhrmester D. The Network of Relationships Inventory: Behavioral Systems Version. Int J Behav Dev. 2009;33(5):470-8.

69. Cohen S, Kamarck T, Mermelstein R. A global measure of perceived stress. J Health Soc Behav. 1983;24(4):385-96.

70. Radloff $L$. The CES-D scale: a self report depression scale for research in the general population. Appl Psychol Meas. 1977;1(3):385-401.

71. Logsdon MC, Usui WM, Nering M. Validation of Edinburgh Postnatal Depression Scale for adolescent mothers. Archives of Womens Mental Health. 2009;12(6):433-40.

72. Cox JL, Holden JM, Sagovsky R. Detection of postnatal depression: development of the 10-item Edinburgh Postnatal Depression Scale. Br J Psychiatry. 1987;150:782-6.

73. Sheehan DV, Sheehan KH, Shytle RD, Janavs J, Bannon Y, Rogers JE, et al. Reliability and validity of the Mini International Neuropsychiatric Interview for Children and Adolescents (MINI-KID). J Clin Psychiatry. 2010;71(3):313-26.

74. Cooper Z, Cooper PJ, Fairburn CG. The validity of the Eating Disorder Examination and its subscales. Br J Psychiatry. 1989;154(6):807-12.

75. Cooper Z, Fairburn C. The Eating Disorder Examination: a semistructured interview for the assessment of the specific psychopathology of eating disorders. Int J Eat Disord. 1987;6(1):1-8.

76. Tanofsky-Kraff M, Theim KR, Yanovski SZ, Bassett AM, Burns NP, Ranzenhofer LM, et al. Validation of the emotional eating scale adapted for use in children and adolescents (EES-C). Int J Eat Disord. 2007;40(3):232-40.

77. Francis SL, Letuchy EM, Levy SM, Janz KF. Sustained effects of physical activity on bone health: lowa Bone Development Study. Bone. 2014;63:95-100.

78. Evenson KR, Catellier DJ, Gill K, Ondrak KS, McMurray RG. Calibration of two objective measures of physical activity for children. J Sports Sci. 2008;26(14): 1557-65.

79. Arora T, Broglia E, Pushpakumar D, Lodhi T, Taheri S. An investigation into the strength of the association and agreement levels between subjective and objective sleep duration in adolescents. PLoS One. 2013;8(8):e72406.

80. Tryon WW. Nocturnal activity and sleep assessment. Clin Psychol Rev. 1996; 16(3):197-213.

81. Ogden CL, Kuczmarski RJ, Flegal KM, Mei Z, Guo S, Wei R, et al. Centers for Disease Control and Prevention 2000 growth charts for the United States: improvements to the 1977 National Center for Health Statistics version. Pediatrics. 2002;109(1):45-60.

82. Misra M, Bredella MA, Tsai P, Mendes N, Miller KK, Klibanski A. Lower growth hormone and higher cortisol are associated with greater visceral adiposity, intramyocellular lipids, and insulin resistance in overweight girls. American Journal of Physiology-Endocrinology and Metabolism. 2008;295(2):E385-E92.

83. Bosch TA, Dengel DR, Kelly AS, Sinaiko AR, Moran A, Steinberger J. Visceral adipose tissue measured by DXA correlates with measurement by $C T$ and is associated with cardiometabolic risk factors in children. Pediatr Obes. 2015; 10(3):172-9.

84. Yeckel CW, Weiss R, Dziura J, Taksali SE, Dufour S, Burgert TS, et al. Validation of insulin sensitivity indices from oral glucose tolerance test parameters in obese children and adolescents. J Clin Endocrinol Metab. 2004;89(3):1096-101.

85. Dalla Man C, Campioni M, Polonsky KS, Basu R, Rizza RA, Toffolo G, et al. Two-hour seven-sample oral glucose tolerance test and meal protocol: minimal model assessment of beta-cell responsivity and insulin sensitivity in nondiabetic individuals. Diabetes. 2005;54(11):3265-73.

86. Ellis KJ, Yao M, Shypailo RJ, Urlando A, Wong WW, Heird WC. Bodycomposition assessment in infancy: air-displacement plethysmography compared with a reference 4-compartment model. Am J Clin Nutr. 2007; 85(1):90-5.

87. Clatworthy J. The effectiveness of antenatal interventions to prevent postnatal depression in high-risk women. J Affect Disord. 2012;137(1-3):2534.

88. Dimidjian S, Goodman SH, Sherwood NE, Simon GE, Ludman E, Gallop R, et al. A pragmatic randomized clinical trial of behavioral activation for depressed pregnant women. J Consult Clin Psychol. 2017;85(1):26-36.

89. O'Mahen H, Himle JA, Fedock G, Henshaw E, Flynn H. A pilot randomized controlled trial of cognitive behavioral therapy for perinatal depression adapted for women with low incomes. Depress Anxiety. 2013;30(7):679-87.

90. Lenze SN, Potts MA. Brief Interpersonal Psychotherapy for depression during pregnancy in a low-income population: a randomized controlled trial. J Affect Disord. 2017;210:151-7.
91. Glasofer DR, Tanofsky-Kraff M, Eddy KT, Yanovski SZ, Theim KR, Mirch MC, et al. Binge eating in overweight treatment-seeking adolescents. J Pediatr Psychol. 2007;32(1):95-105.

92. Muktabhant B, Lawrie TA, Lumbiganon P, Laopaiboon M. Diet or exercise, or both, for preventing excessive weight gain in pregnancy. Cochrane Database Syst Rev. 2015;6:CD007145.

93. Whitehead AL, Julious SA, Cooper CL, Campbell MJ. Estimating the sample size for a pilot randomised trial to minimise the overall trial sample size for the external pilot and main trial for a continuous outcome variable. Stat Methods Med Res. 2016;25(3):1057-73.

94. Cocks K, Torgerson DJ. Sample size calculations for pilot randomized trials: a confidence interval approach. J Clin Epidemiol. 2013;66(2):197-201.

95. Eldridge SM, Chan CL, Campbell MJ, Bond CM, Hopewell S, Thabane L, et al. CONSORT 2010 statement: extension to randomised pilot and feasibility trials. BMJ. 2016;355:15239.

96. Okun ML, Tolge M, Hall M. Low socioeconomic status negatively affects sleep in pregnant women. J Obstet Gynecol Neonatal Nurs. 2014;43(2):1607.

97. Hurley KM, Caulfield LE, Sacco LM, Costigan KA, Dipietro JA. Psychosocial influences in dietary patterns during pregnancy. J Am Diet Assoc. 2005; 105(6):963-6.

98. Gentile S. Untreated depression during pregnancy: short- and long-term effects in offspring. A systematic review. Neuroscience. 2017;342:154-66.

99. Mutamba BB, Kane JC, de Jong J, Okello J, Musisi S, Kohrt BA. Psychological treatments delivered by community health workers in low-resource government health systems: effectiveness of group interpersonal psychotherapy for caregivers of children affected by nodding syndrome in Uganda. Psychol Med. 2018;48(15):2573-83.

\section{Publisher's Note}

Springer Nature remains neutral with regard to jurisdictional claims in published maps and institutional affiliations.

Ready to submit your research? Choose BMC and benefit from:

- fast, convenient online submission

- thorough peer review by experienced researchers in your field

- rapid publication on acceptance

- support for research data, including large and complex data types

- gold Open Access which fosters wider collaboration and increased citations

- maximum visibility for your research: over $100 \mathrm{M}$ website views per year

At BMC, research is always in progress.

Learn more biomedcentral.com/submissions 